\title{
Holographic Technology Applied in Modern Advertising Design Innovative Thinking
}

\author{
Gao Tong
}

Tianjin University of Technology, Tianjin 300384, China

Keywords: holographic image; holographic technology; modern advertising design

\begin{abstract}
The rise of holographic technology is a revolution in modern advertising design. The wave of change has taken shape in many aspects such as structure, form and performance. The technology breaks the traditional advertising design concept and enables audiences to interact in person to feel the shock of super-senses, which is undoubtedly the development of modern advertising design trends and requirements. This paper starts with the development history of holographic technology, takes the combined case of this technology and modern advertisement design as the research background, conducts the omni-directional thinking from the application status, application analysis and application prospect, analyzes and summarizes this technology in simple terms In the modern advertising design application situation, which is forward-looking for the convergence of future holographic technology and modern advertising design.
\end{abstract}

\section{Introduction}

Modern advertising design gradually evolves from the traditional static design to the interactive design pattern. The emergence of holographic technology has provided a new space for design and display of modern advertising. For example, on June 22, 2013, "holographic projection test window" appeared in the flagship store of Hongxing Erke at Qianmen Street in Beijing. It is the emerging holographic technology that moves the traditional fitting room to the window. This unprecedented display mode allows consumers to intuitively feel the details of the product features and the actual wearing effect, marketing value can not be underestimated, we can see the holographic technology in the modern advertising design of extraordinary significance. First of all, holographic technology has broken the traditional media constraints, its strong sense of space, perspective, high definition, contrast and color reproduction make advertising design more real three-dimensional. In fact, holographic technology used in modern advertising design, products in the most unique way to show in front of and interact with the audience, this modern and stylish interaction to consumers an unprecedented interactive experience process. The holographic technology will be introduced into the advertising field and applied to promote a good interactive and realistic experience will be the trend of modern advertising design, the people's heart.

\section{Holographic Technology in New Media Era}

\subsection{The origin of the new media era}

The term "new media" first became popular in American society. In 1967, P. Goldmark, then director of the CBS Institute of Technology in the United States, first proposed the term "new media" in the development of EVR's merchandise program, The unstoppable trend spreads all over the world. The concept of "new" in "new media" is relative and is constantly evolving. The new media era is a product of the rapid development of science and technology. Compared to traditional media such as television, radio, newspapers and periodicals, the new media era is based on mobile technology, network technology and digital technology, and is based on electronic equipment such as digital television, mobile phones and computers, The satellite, wireless communications network, the Internet and other channels as the means of entertainment and information supply media age [2].

With the rapid development of science and technology, exchanges within the society have 
increased, interdependence has been intensified, and the spread of information has rapidly and widely shortened the distance between space and time. Globally, the mainstream of the development of the times has become informatized. Advances in science and technology have brought new media to the forefront of this era. It has also brought about a rapid expansion of our life while introducing some new world.

\subsection{Generation and principle of holographic technology}

In 1947, holographic technology was first discovered and studied. Dennis Gabor of the Imperial College London proposed the imaging concept of holographic technology and won the 1971 Nobel Prize in Physics. In the late 1960s, Deman et al. Proposed a new holographic concept of digital holography, thus creating a new era of precision holography [3].

Holographic image technology uses the principle of interference and diffraction of light to reflect the amplitude and phase of a specific light wave of an object in the form of interference fringes to reproduce the true three-dimensional image of the object. In a narrow sense, holographic technology refers to scanning a certain area within a certain point, by scanning the information arranged in a single point to form a true 3D image. In a broad sense, holographic technology is an optically advanced cutting-edge technology that perfectly combines information storage and laser technology with the search for sensitive recording media and the right reproduction. As we all know, all the light has a color, direction and brightness of the three properties, whether it is the early silver version of the camera, black and white photos or color photos can only record one of the three attributes, only the holographic technology can capture The three properties of light, the true representation of objects in the true three-dimensional space scene. The technology contains a large amount of data and information of the object, so that the three-dimensional image produced by the object gives the observer an immersive real feeling.

\subsection{Trends of holographic technology}

With the continuous development of holographic technology, the design and manufacturing costs will be reduced, and the amount of information storage will be more and more abundant. This technology will increasingly show the unmistakable image rich colors and flexible viewing angles. Although the current holographic technology has not been perfect yet, as the special effects in the movie show, the visual and sensational effect of the technology has deeply rooted in people's heart and profoundly influenced people's aesthetic concepts.

Holographic technology has been widely used in many aspects of social production and life since it was proposed, developed to be popularized, and now it has been integrated with many disciplines and formed holographic molding, holographic storage and holographic display. For example, in the field of anti-counterfeiting technology, holograms are used as anti-counterfeit labels, holographic maps are applied in the military field, three-dimensional images of the patient's site are provided by using laser holographic imaging in medicine, and multi-zone images are reproduced by using holographic screens in film and television. Using holographic images for product packaging and promotion and more.

\section{The Application Status Quo of Holographic Technology in Modern Advertising Design}

\subsection{The status of foreign applications}

Abroad has many hollywood brand advertising products using holographic technology to display their products, such as 2010 fashion brand Burberry show that the use of holographic technology and models alternately display clothing, the introduction of high-tech fashion show field, so that the audience feel To a different kind of viewing experience [5]; Prada designed the personality fitting system, the use of holographic images show fitting effect; Australian university developed a car in the back seat of the interactive multi-touch device using holographic technology for the audience With gestures "volley" control of virtual scenes; there is the German Volvo cars outdoor advertising and so on. 


\subsection{The status of domestic applications}

At present, the application of holographic technology in the field of advertising is relatively narrow, mainly used in small show cases and the stage. The technology used in advertising design to showcase jewelry, small electronics and corporate identity, content is generally a relatively simple rotating animation. In addition, the 180-degree monolithic hologram on the stage shows mostly live-action holographic effects, virtual shows and virtual and live-action interactions.

\section{3 holographic technology in modern advertising design advantages and obstacles}

\subsubsection{Application advantages}

The emergence and development of holographic technology has greatly opened up the creativity and imagination in the field of modern advertising design, creating a virtual but realistic world for consumers. The birth of this technology and its application in advertising design satisfy consumers' desire and pursuit for a fantasy world, and according to various existing case studies, the technology is quite acceptable among the public, this laid a solid market foundation for the further development and application of holographic technology in modern advertising.

Second, the two-dimensional to three-dimensional and the plane to the three-dimensional conversion is the inevitable trend in the field of advertising design, realistic and complete user experience and the pursuit of advertising design is difficult to value holographic images unique hyper-realistic three-dimensional experience, no doubt for advertising design Dimensional three-dimensional become the trend of the times, fit the times and demands of the new media age.

Thirdly, as the application of holographic technology continues to mature, the combination of this technology with other technologies will also develop and improve day by day. The technical fields of modern advertising design will continue to broaden, and the effect and audience experience will also become increasingly perfect.

\subsubsection{Application obstruction}

While holographic technology has many advantages in modern advertising design, there will be some hurdles in its future publication process. First of all, holographic technology requires very high light and environmental requirements. Specific lighting and environment are the prerequisites for this technology. However, due to the shortcomings of current technologies, the implementation of these requirements requires harsh conditions. Second, while the cutting edge of holographic technology is extremely costly due to the control of individual companies in a few developed countries, this poses some limitations on the development, diffusion and application of this technology. Third, the domestic implementation of new technologies like holography is limited, and the development of this technology lacks a strong support [6].

\section{Application of Holographic Technology in Modern Advertising Design}

\subsection{Holographic technology in modern advertising design concept}

One of the application concepts of holographic technology in modern advertising design is the omni-directional sensory experience. Consumers are increasingly seeking new and chic, stimulating senses as the basic principle to attract the audience. Holographic technology can make the audience enjoy the immersive Impressions and feelings, this is the technology beyond the traditional advertising design unique. The second application concept is a three-dimensional interactive experience. The holographic technology enables consumers to simultaneously experience multiple applications of text, pictures and sound effects. The potential needs and internal needs of the audience are correspondingly raised to produce positive buying behavior. The technology is widely used in new media the era of product promotion is also the significance of this.

\subsection{Holographic technology in modern advertising design application form}

Holographic technology into the field of modern advertising design shorter time, although the application of this emerging technology in this area is still in the development and exploration stage, 
but its application form is more clear.

First, the holographic technology has excellent lighting performance in a closed environment due to its high light requirements for the surrounding environment. Second, the holographic technology can achieve variability and dynamic advertising effects, displaying the advertising image in different spatial locations under the conditions of moving the projection equipment and transforming the output signals, thereby bringing the audience the surrealistic magical feeling. Third, the application of holographic technology requires other technical support. The interactive sensing technology and light sensing technology are also emerging science and technology in the new media era. By combining them with holographic technology, audiences can have more initiative in advertising, you can also expand the three-dimensional advertising, rich design content. Fourth, the main body of advertising design is always the commodity. In the whole process, the application of holographic technology should emphasize the design principle of commodities. Designers should fully consider this point, making the highlight of the technology not be overwhelming. Fifth, the holographic technology presented to the audience is a kind of virtual image, with complex production processes and design standards behind the display. Therefore, advertising design should take full account of the coordination of the two when applying the technology [7].

\subsection{Holographic technology in modern advertising design application effect}

Generally speaking, there is a step-by-step process from the beginning of the purchase desire of the product to the actual purchase of the product. First, the purchase demand is generated, secondly, the purchase target is determined, and then the purchase decision is made, and then the purchase and use of the product are performed, and finally, User experience evaluation product information feedback. Consumers are mainly motivated by subjective feelings when they generate purchase demand, ie, tactile, visual, auditory and etc. This is the most direct response of consumers to external information and the primary point of understanding, using, experiencing and changing the object [8]. Holographic technology can display three hundred and sixty degrees of dynamic images, stereoscopic images and music effects in advertisements. The multi-directional information input of consumers effectively brings the audience into the real atmosphere of feeling the goods and effectively deepens the consumption Impressions of the advertisement. In addition, holographic technology is different from the traditional form of advertising design, which can be both real and performance of the effective combination of the audience in the interactive experience of reality.

\subsection{Holographic technology in modern advertising design value and application prospects}

First, the value of reflection

In the global trend of advertising design, more advertising design will continue to improve the means and methods will use holographic technology to play an unprecedented charm. The annual International Conference on Holographic Technology will present stunning three-dimensional holographic images that are stunning and reverie of realistic stereo vision. 20th century advertising design is based on visual elements, and the 21st century design must be combined with the sense of smell, touch, temperature and weight, etc., holographic technology to meet this development needs.

Second, the application prospects

Holography is set to grow rapidly in the future, moving towards greater dynamics and interactivity. Its imaging is no longer confined to the existing aerial imaging. Instead, it is expected to develop into a semi-transparent ultrathin particle concentration screen imaging that gives viewers the possibility and space to walk freely on the screen [9]. This is the interactive experience of holographic technology that spans space constraints, creating an unprecedented full-scale stereoscopic space that allows viewers to engage and interact with 3D images at zero distances. This can be described as a video revolution through time and space. Because of the new interactive concept of holographic technology, the interaction between time and space and the unity of perception are the new experiences of inner emotion and subjective thinking experienced by the viewer in this virtual interactive environment a more humane interactive experience. Imagine in the future online shopping process, consumers are no longer seen in kind shooting two-dimensional photos, but multi-angle, all-round like three-dimensional images of the kind. In the new BMW 
concept car showroom, the car's images are suspended in the air, the viewer can clearly see every detail, you can touch the finger through the concept car full rotation and three-dimensional decomposition, or even in the the audience immediately turned into an airplane. With the progress of holographic technology and the improvement of the holographic imaging quality, the technology of the future society will be applied to all aspects of people's life more widely.

\section{Conclusion}

Holographic technology is the product of modern science and technology, the introduction of this technology in modern advertising can be artistic, scientific and decorative design integration, advertising design performance can be exchanged between reality and fantasy, bringing the audience a new design experience. Advertising is a combination of aesthetic and practical, its purpose is to pass information as consumers, to bring profits to businesses. In the perfect combination of modern technology and art aesthetics, immersive experiences and perceptions allow consumers to switch from passive information acceptance to active information access. At the same time, however, the mastery of technology, the combination of specialization, and the reserve of qualified personnel will all be challenges in the application of holographic technology to modern advertising design.

\section{References}

[1] SHE Lin-zi.Application of 3D Holographic Projection Technology in Display Design [J]. Modern decoration, 2016, (8): 22.

[2] Cui Lu, Yang Junshun.Analysis of holographic imaging technology and its application [J] .America and era (first half), 2009, (9): 115-117.

[3] Jin Xiaofei. Holographic imaging as a new media art language research [D]. Dalian University of Technology, 2014.

[4] Guo Yan. The new interactive concept of holographic imaging technology [D]. Shenyang University of Aeronautics and Astronautics, 2014.

[5] XU Xu-ming.Analysis on the Influence of Holographic Imaging Technology on Digital Media Art Design [J] .Artworks and Technology, 2016, (7): 411-412.

[6] Ge Fei, Mo Yanfeng.Analysis of the impact of 3D holographic projection technology on modern advertising and design specialty [J]. Art Education, 2016, (5): 198-199.

[7] Guo Wen. Probe into holographic and modern art design [J]. Modern decoration (Theory), 2016, (3): 200.

[8] Wang Yueling. Holographic imaging to create "three-dimensional news" - holographic imaging technology in the field of media [J] Chinese Journalists, 2014, (9): 126-127.

[9] Hu Ze, King Yan. Holographic imaging in international news [J]. Science and Technology of Communications, 2014, (22): 139, 190. 